ROSANGELA MONTEIRO

\title{
Avaliação das alterações anatômicas cardíacas secundárias ao enfisema pulmonar: estudo experimental em ratos
}

Tese apresentada ao Departamento de Cardio-Pneumologia da Faculdade de Medicina da Universidade de São Paulo para obtenção do título de Doutor em Ciências.

Área de concentração: Cirurgia Torácica e Cardiovascular

Orientador: Prof. Dr. Fabio Biscegli Jatene

São Paulo

2004 
"Não confunda jamais conhecimento com sabedoria.

Ulm o ajuda a ganfar a vida; o outro a construir uma vida" S. Carey 


\section{DEDICATÓRIA}


Aos meus pais e irmãos,

por tudo que fizeram por mim e pelo que representam em minfla vida...

... pelo exemplo, pelo carinfo, pelo colo, pelo amor em todos os momentos, ... por vocês existirem. 
Ao Prof. Dr. Fabio Biscegli gatene,

com que m tenfo tido o privilégio de trabalhar há quase 15 anos... por ter me ensinado os primeiros passos na carreira científica por me apoiar nos de mais...

Obrigada por ser meu orientador, meu chefe, meu amigo...

... por estar presente nos momentos mais importantes e também nos mais difice is da minfra vida... 
AGRADECIMENTOS 
Ao Prof. Dr. Noedir Antônio Groppo S tolf, coordenador da pós-graduação na Áre a de Cirurgia Torácica e Cardiovascular da Faculdade de Medicina da Universidade de $S$ ão Paulo, por me acolher como primeiro profissional não-médico para realização de Doutorado neste programa. Agradeço pela confiança em mim de positada, proporcionando-me a fonrosa oportunidade de desenvolver esta tese.

Ao Prof. Dr. Paulo Manuel Pêgo-Fernandes, pelo apoio e incentivo constantes na minha atividade acadêmica pelas valiosas sugestões no planejamento deste estudo.

Ao $\operatorname{Prof}$. Dr. Luís Felipe Pinho More ira, pela ate nção dispensada durante a pós-graduação e pelo exemplo de rigor científico e zelo pela pesquisa.

Ao Prof. Dr. Pedro Primo Bombonato e ao Dr. Alexandre Ciappina Hue 6 pelas importantes sugestões na análise do "boneco de tese" apresentado para exame de qualificação, as quais abriram perspectivas para realização de novos estudos em continuidade a esta tese.

Aos amigos Rogério Pazetti, Aristides Tadeu Correia, Dolores Helena Rodrigues Ferreira Rivero e Luiza Antônia Manoel, pela competência e profissionalismo demonstrados durante a realização dos experimentos que resultaram nesta tese.

Ao $\mathcal{D r}$. Wanderley Marques Bernardo, me urande amigo e companthe iro de todas as horas, por aguçar o meu espirito critico, dividir seus confecimentos de metodologia cientifica e, acima de tudo, por sua amizade incondicional.

Ao Dr. Miguel Lia $\mathcal{T} e d d e$, pelo carinho, pelo estimulo e pela incansável disposição em ajudar.

Aos meus amigos do Laboratório Anatômico Cirúrgico do Instituto do Coração, Argemiro Falcetti J unior, Eliana Ogata e Sérgio Spezzia, pelo companteiris mo e de dicação sempre demonstrados durante esses anos de convivência.

Às minhas amigas "super-poderosas", Ana Cristina Martins Monteiro, Luciana Azevedo Luppi e Sueli Vieira Santos, pela "gasolina" nos 
momentos em que achei que ela fosse acabar, pelacerteza de que sempre posso contar com vocês.

$\mathcal{A} \mathcal{N}$ (e usa Rodrigues Dini, I uliana Lattari Sobrinho e Eva Malheiros Guis de Oliveira, funcionárias da Coordenaria de Pós-graduação do Instituto do Coração, por me incentivarem e estarem sempre prontas a ajudar na superação das dificuldades encontradas.

À Creusa Maria Rovieri Dal Bó, pelo auxilio na realização da análise estatistica deste trabalho.

Ao Prof. Dr. Paulo Hilário $\mathcal{N}$ ascimento Saldiva e à farmacêutica Cíntia $G$. C. do Nascimento Freire, pela realização da gasome tria do sangue arterial na Divisão de Laboratório Central do Hospital das Clínicas da Faculdade de Medicina da Universidade de São Paulo.

$\mathcal{A}$ Yon Morato, pela atencios a revisão e ade quação do summary.

Aos colegas do curso de pós-graduação, pela agradável convivência. 
SUMÁRIO 


\section{SUMÁRIO}

Resumo

Summary

1 INTRODUÇÃO 1

1.1 Doença Pulmonar Obstrutiva Crônica e Enfisema Pulmonar 2

1.2 Modelos Animais de Doença 6

1.3 Modelos Experimentais de Enfisema Pulmonar 8

1.4 Alterações Cardíacas Secundárias ao Enfisema Pulmonar 11

2 OBJETIVOS 13

3 MATERIAL E MÉTODO 15

3.1 Aspectos Éticos 16

$\begin{array}{ll}3.2 & \text { Estudo Piloto } \\ 3.3 & \text { Protocolo Expermental }\end{array}$

3.3 Protocolo Experimental 17

$\begin{array}{lll}\text { 3.3.1 Grupos Experimentais } & 17\end{array}$

$\begin{array}{lll}3.3 .2 & \text { Anestesia } & 18\end{array}$

3.3.3 Ventilação Mecânica 19

3.3.4 Indução de Enfisema Pulmonar por Papaína 20

3.3.5 Manutenção dos Animais 21

3.3.6 Sacrifício 22

3.4 Parâmetros Analisados 23

3.4.1 Avaliação Sangüínea: Gasometria do Sangue Arterial 23

3.4.2 Avaliação Pulmonar: Análise Histopatológica e Morfométrica para Determinação do Enfisema Pulmonar 24

3.4.3 Avaliação do Peso Corpóreo e de sua Relação com o Peso Cardíaco 27

3.4.4 Avaliação Cardíaca: Análise Morfométrica dos Corações 28

3.5 Análise Estatística 32

4 RESULTADOS 33

4.1 Mortalidade $\quad 34$

4.2 Avaliação Sangüínea: Gasometria do Sangue Arterial 35

4.2.1 Saturação de Oxigênio 35

4.2.2 Pressão Parcial de Oxigênio 36

4.2.3 Pressão Parcial de Gás Carbônico 38

4.3 Avaliação Pulmonar: Análise Histopatológica e Morfométrica para Determinação do Enfisema Pulmonar 40

4.4 Avaliação do Peso Corpóreo e de sua Relação com o Peso Cardíaco

4.4.1 Peso Corpóreo ao Sacrifício 43

4.4.2 Peso Cardíaco 45

4.4.3 Relação entre o Peso Corpóreo e o Peso Cardíaco 46 
4.5 Avaliação Cardíaca: Análise Morfométrica dos Corações 48

4.5.1 Espessura da Parede do Ventrículo Direito 48

4.5.2 Espessura da Parede do Septo Interventricular 49

4.5.3 Espessura da Parede do Ventrículo Esquerdo 50

4.5.4 Área da Cavidade Ventricular Direita 52

4.5.5 Área da Cavidade Ventricular Esquerda 53

5 DISCUSSÃO 56

5.1 Considerações Gerais $\quad 57$

5.2 Análise do Material e Método 59

5.2.1 Escolha do Rato como Animal de Experimentação 59

5.2.2 Escolha do Modelo de Indução de Enfisema com Papaína 60

5.3 Análise dos Resultados Obtidos 63

5.3.1 Avaliação Sangüínea: Gasometria do Sangue Arterial 63

5.3.2 Avaliação Pulmonar: Análise Histopatológica e Morfométrica para Determinação do Enfisema Pulmonar 64

5.3.3 Avaliação do Peso Corpóreo e de sua Relação com o Peso Cardíaco 66

5.3.4 Avaliação Cardíaca: Análise Morfométrica dos Corações 67

5.4 Perspectivas Futuras 71

6 CONCLUSÕES

7 REFERÊNCIAS 


\section{RESUMO}




\section{RESUMO}

Monteiro R. Avaliação das alterações anatômicas cardíacas secundárias ao enfisema pulmonar: estudo experimental em ratos [tese]. São Paulo: Faculdade de Medicina, Universidade de São Paulo; 2004. 87p.

Introdução: Além das já conhecidas repercussões morfofuncionais que ocorrem nos pulmões de pacientes com enfisema pulmonar, vários órgãos e estruturas sofrem o impacto dessas alterações, dentre eles o coração. Apesar da extensa literatura sobre enfisema pulmonar, há poucos trabalhos avaliando os efeitos do enfisema induzido experimentalmente sobre o coração, sobretudo cronicamente.

Objetivo: Este trabalho tem como objetivo avaliar a ocorrência e as repercussões de enfisema pulmonar quimicamente induzido e as alterações morfológicas presentes em corações de ratos após sua indução, acompanhando sua progressão ao longo do tempo.

Material e Método: Foram avaliados 75 ratos divididos em dois grupos, papaína $(\mathrm{N}=50)$ e controle $(\mathrm{N}=25)$, submetidos à instilação intratraqueal de papaína e solução fisiológica, respectivamente. Os animais foram sacrificados $30,60,90,120$ ou 180 dias após a instilação. Foi realizada gasometria do sangue arterial, análise morfométrica dos pulmões e coração, bem como avaliação do peso corpóreo e sua relação com o peso cardíaco .

Resultados:A instilação de papaína produziu destruição do tecido alveolar, mimetizando alterações morfológicas encontradas no enfisema pulmonar, sem repercussão na função de trocas gasosas. O ventrículo direito e septo interventricular não apresentaram alterações macroscópicas significativas no período de até seis meses após a indução do enfisema. A cavidade do ventrículo direito apresentou dilatação, a partir de 120 dias após indução do enfisema. O ventrículo esquerdo apresentou significativa redução da área de sua cavidade, 90 dias após a indução do enfisema pulmonar, acompanhada de discreto espessamento de sua parede.

Conclusões: O modelo experimental empregado foi eficiente para induzir morfologicamente 0 enfisema pulmonar, embora sem alterações hemogasométricas. A presença de enfisema pulmonar não provocou alterações morfológicas na parede do ventrículo direito e septo interventricular. A destruição alveolar induziu hipertrofia do ventrículo esquerdo e dilatação do ventrículo direito provavelmente secundária a esta.

Descritores: Modelos animais de doenças. Ratos Wistar. Papaína/farmacologia. Enfisema pulmonar/induzido quimicamente. Enfisema pulmonar/fisiopatologia. Ventrículo cardíaco/anatomia \& histologia. 
SUMMARY 


\section{SUMMARY}

Monteiro R. Evaluation of the cardiac anatomical alterations secondary to the pulmonary emphysema: experimental study in rats. [thesis]. São Paulo: Faculdade de Medicina, Universidade de São Paulo; 2004. 87p.

Introduction: Besides the well known morphofunctional repercussions observed in patients lungs with pulmonary emphysema, several organs and structures suffer the alterations impact, the heart among them. In spite of the vast pulmonary emphysema literature, there are few papers analyzing the effects of the experimentally induced emphysema in the heart, above all chronically.

Objective: The purpose of this study is to evaluate the occurrence and repercussions of the pulmonary emphysema chemically induced and the morphological alterations present in heart of rats post-induction, following its progression afterwards.

Material and Method: Seventy five rats divided into two groups papain $(\mathrm{N}=50)$ and control $(\mathrm{N}=25)$, submitted to intratracheal instillation of papain and saline solution, respectively, were evaluated. The animals were sacrificed 30,60 , 90,120 or 180 days post-instillation. Arterial blood gases, cardiac and pulmonary morphometrical analysis as well as body weight and its relation to cardiac weight evaluation were performed.

Results: The papain instillation produced alveolar tissue destruction similar to the morphological alterations observed in pulmonary emphysema without arterial blood gases changes. Right ventricle and interventricular septum did not show significant macroscopic alterations until six months after emphysema induction. The right ventricle area presented enlargement 120 days post-induction of the pulmonary emphysema. The left ventricle presented significant cavity area decrease 90 days post-induction of the pulmonary emphysema, followed by slight wall thickening.

Conclusions: The adopted experimental model was efficient to morphologically induce the pulmonary emphysema, although, without arterial blood gases changes alterations. The pulmonary emphysema presence did not provoke morphological changes in the right ventricle wall and interventricular septum. The alveolar destruction induced left ventricular hypertrophy and enlargement of the right ventricle, probably secondary to the first one.

Keywords: Disease models, animal. Rats, Wistar. Pulmonary emphysema/chemically induced. Pulmonary emphysema/physiopathology. Heart ventricle/anatomy \& histology. 